\title{
Study of Resistive Superconducting Transition of Bulk $\left(\mathrm{Bi}_{0.6} \mathrm{~Pb}_{0.4}\right)_{2} \mathrm{Sr}_{2} \mathrm{Ca}_{2} \mathrm{Cu}_{3} \mathrm{O}_{x}$
}

\author{
W.M. Woch*, M. Chrobak, R. Zalecki, A. Kolodziejczyk \\ AGH University of Science and Technology, Faculty of Physics and Applied Computer Science, \\ Solid State Physics Dept., Al. Mickiewicza 30, 30-059 Kraków, Poland
}

\begin{abstract}
The temperature widths of the resistive transition as well as the thermal fluctuations of bulk $\left(\mathrm{Bi}_{0.6} \mathrm{~Pb}_{0.4}\right)_{2} \mathrm{Sr}_{2} \mathrm{Ca}_{2} \mathrm{Cu}_{3} \mathrm{O}_{x}$ superconductor with $T_{c, 50 \%}=107.1 \mathrm{~K}$ were studied. The applied magnetic field widens the resistive transition according to the following formula: $\Delta T=C H^{m}+\Delta T_{0}$. The value of exponent $m=0.44$ suggests that there is strong pining of vortices, especially at lower temperatures. The critical exponents $\lambda$ of the conductivity were calculated using the following equation: $\Delta \sigma=K \varepsilon^{-\lambda}$, with the $\lambda_{1}=1.75$ close to the zero critical temperature and $\lambda_{2}=3.77$ at higher temperatures. These values were discussed within the available theoretical models.
\end{abstract}

DOI: $10.12693 /$ APhysPolA.126.328

PACS: 74.25.F-; 74.25.Uv; 74.25.Wx; 74.72.-h; 64.60.F-

\section{Introduction}

In high temperature superconductors (HTS) a combination of the short coherence length, large anisotropy, large magnetic penetration depth and high critical temperatures make the fluctuation effects many orders of magnitude larger than in the conventional superconductors. In consequence the resistive transition from normal to superconducting state shows large and anomalous broadening, even without a magnetic field. In this paper the broadening of the resistive transitions and thermal fluctuations of ceramic $\left(\mathrm{Bi}_{0.6} \mathrm{~Pb}_{0.4}\right)_{2} \mathrm{Sr}_{2} \mathrm{Ca}_{2} \mathrm{Cu}_{3} \mathrm{O}_{x}$ superconductor have been studied.

\section{Experimental details}

The sample of the ceramic bismuth-based 2223 superconductor, partially substituted with $\mathrm{Pb}$ for $\mathrm{Bi}$ atoms, was prepared by the standard solid state reaction procedure that was well described in the literature [1]. The $\mathrm{X}$-ray diffraction showed that there are two superconducting phases: the dominant 2223 phase with $T_{c, 50 \%}=$ $107.1 \mathrm{~K}$ and the minor 2212 phase with $T_{c}=90 \mathrm{~K}$. The measurements of the resistance versus temperature and magnetic field were carried out using the four probes a.c. method. The electrical contacts were made by Leitsilber 200 silver paint and after heat up to $300{ }^{\circ} \mathrm{C}$ their resistances were less than $0.5 \mathrm{Ohms}$. The sample was placed in the center of copper coils, which produced the d.c. magnetic field. The temperature was monitored by a Lake Shore temperature controller. The Stanford SR 830 lock-in nanovoltmeter served both as a source for a.c. currents with a frequency of $188 \mathrm{~Hz}$ and amplitudes up to $100 \mathrm{~mA}$ and as a voltage meter.

\section{Results and discussion}

The temperature dependencies of the resistance for selected values of the applied d.c. magnetic fields are shown

*corresponding author; e-mail: wmwoch@agh.edu.pl in Fig. 1. One can observe there a significant widening of the transition in consequence of applying of the external magnetic field, which is typical for HTS, especially for thallium based superconductors $[2,3]$. This phenomenon has been interpreted in the frame of the vortex structure and flux motion mechanism $[4,5]$.

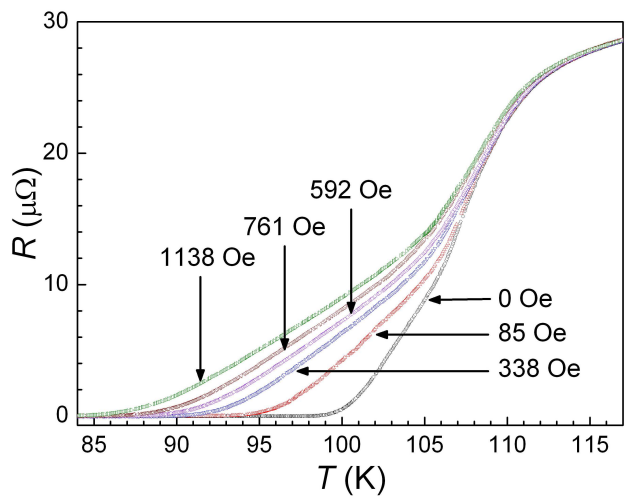

Fig. 1. Magnetoresistance as a function of temperature.

The widths of the resistive transitions $\Delta T=T_{90 \%}-$ $T_{10 \%}$ (between $10 \%$ and $90 \%$ of the normal state resistance, just above the onset temperature) versus the applied magnetic field are shown in Fig. 2. For HTS the resistive transition already for $H=0$ is relatively wide. The widths of the resistive transitions may be described by the following form [2]:

$$
\Delta T=C H^{m}+\Delta T_{0},
$$

where $m=2 / 3$ and $\Delta T_{0}$ means the width of the resistive transition at zero d.c. applied magnetic field. The coefficient $C$ depends on the critical current at zero magnetic field and on the critical temperature. The experimental data presented in Fig. 2 were fitted using Eq. 1. There were two fit parameters: $m$ and $C$. The width of the resistive transition $\Delta T_{0}=11.0 \mathrm{~K}$ was taken from the 
experiment (see Fig. 1). The parameters $m$ and $C$ obtained from the fitting procedure are $0.440 \pm 0.008$ and $0.41 \pm 0.03$, respectively. One can notice that at zero applied magnetic field the width of the resistive transition is relatively large and rises with the magnetic fields especially at their lower values.

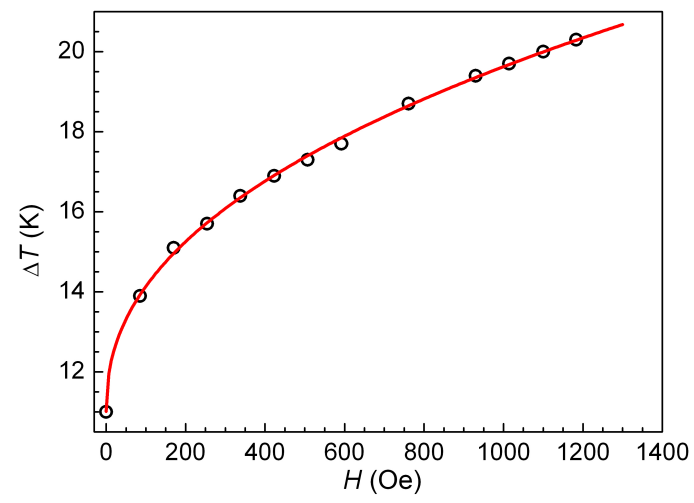

Fig. 2. Width of the resistive transition (open circles). Solid line is the fit by Eq. 1 .

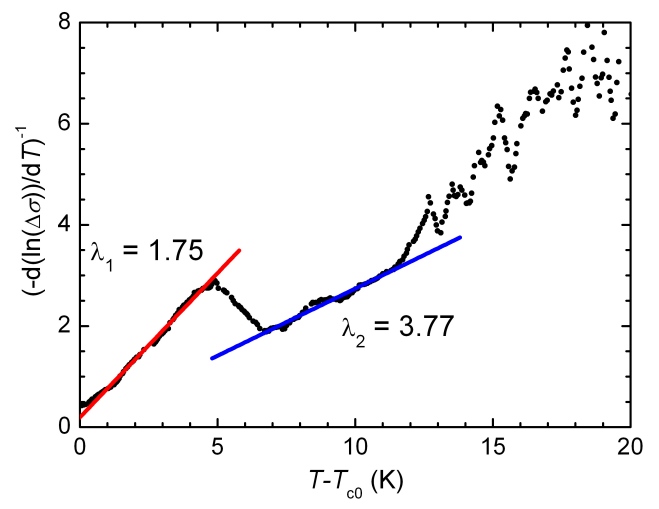

Fig. 3. Plot of $(-d(\ln (\Delta \sigma)) / d T)^{-1}$ versus $T$ (closed circles). Solid lines are the linear fit to linear parts of this plot.

The critical exponents $\lambda$ of the conductivity were calculated using the following equation [6]:

$$
\Delta \sigma=K \varepsilon^{-\lambda},
$$

where $\varepsilon=\left(T-T_{c}\right) / T_{c}$ and $K$ is a constant. To determine the critical exponents we plotted the temperature dependence of the following expression:

$$
-\left[\frac{d}{d T} \ln \Delta \sigma(T)\right]^{-1}=\frac{1}{\lambda}\left(T-T_{c}\right),
$$

where $\Delta \sigma(T)$ is defined by the Eq. 2 . The critical exponent is a reversal slope of the dependence within a linear regions of the temperature. The relation expressed by Eq. 3 is shown in Fig. 3. The calculated critical exponent close to the zero critical temperature was determined to be $\lambda_{1}=1.75 \pm 0.05$. The critical exponent in the temperature interval from $T_{c, 50 \%}$ to the onset temperature is $\lambda_{2}=3.77 \pm 0.07$.

These large values of the critical exponents are difficult to explain and understand, especially, from the point of view of Aslamazov and Larkin approach [6], in which the critical exponents are usually lower than unity. Such interesting behavior is a challenge and an inspiration for the further study.

\section{Conclusions}

The results of this paper can be summarized as follows:

1. The applied magnetic field widens the resistive transition according to the formula (1). This formula fits the experimental data very well. A higher value of the exponent $m=0.44$ suggests that there is rather strong pining of vortices, especially at lower temperatures.

2 . The critical exponents $\lambda$ of the conductivity were calculated using the Eqs. 2, 3. The calculated critical exponent, close to the zero critical temperature, was found to be $\lambda_{1}=1.75$. The critical exponent in the temperature interval from $T_{c, 50 \%}$ to the onset temperature is $\lambda_{2}=3.77$. These large values of the critical exponents are difficult to explain by the known model [6].

\section{Acknowledgments}

This work was supported by the Polish Ministry of Science and Higher Education and its grants for Scientific Research. We appreciate to Dr. Ł. Gondek for the XRD measurements. One of us (M. Ch.) has been partly supported by the EU Human Capital Operation Program, Polish Project No. POKL.04.0101-00-434/08-00.

\section{References}

[1] see for instance: B. Job, M. Rosenberg, Physica $C$ 172, 391 (1991) and references therein.

[2] W.M. Woch, R. Zalecki, A. Kołodziejczyk, O. Heiml, G. Gritzner, Physica C 434, 17 (2006).

[3] W.M. Woch, R. Zalecki, A. Kołodziejczyk, H. Sudra, G. Gritzner, Supercond. Sci. Technol. 21, 085002 (2008).

[4] T.T.M. Plastra, B. Batlogg, L.F. Schneemeyer, J.V. Waszczak, Phys. Rev. Lett. 61, 1662 (1988).

[5] W.K. Kwok, U. Welp, G.W. Crabtree, K.G. Vandervoort, R. Hulscher, J.Z. Liu, Phys. Rev. Lett. 64, 966 (1990).

[6] L.G. Aslamazov, A.I. Larkin, Sov. Phys. Solid State 10, 875 (1968); A.I. Larkin, A. Varlamov, in $S u$ perconductivity, eds. K.H. Bennemann, J.B. Ketterson, vol. 1, p. 369, Springer-Verlag Berlin, Heidelberg, 2008; C.J. Lobb, Phys. Rev. B 36, 3930 (1987). 